\title{
Stabilizability Conditions for Switched Linear Systems with Constant Input via Switched Observer
}

\author{
Takuya SOGA ${ }^{1}$, Naohisa OTSUKA ${ }^{2}$ \\ ${ }^{1}$ Graduate School of Advanced Science and Technology, Tokyo Denki University, \\ Hatoyama-Machi, Hiki-Gun, Saitama, 350-0394, Japan \\ ${ }^{2}$ Division of Science, School of Science and Engineering, Tokyo Denki University, \\ Hatoyama-Machi, Hiki-Gun, Saitama, 350-0394, Japan, \\ otsuka@mail.dendai.ac.jp
}

Abstract: In this paper, stabilizability conditions for switched linear systems with constant input via two types of switched rule which depends on the state of switched observer are presented. The obtained results provide stabilizability conditions via state feedback switched rule. Further, two illustrative numerical examples are also given.

Keywords: Switched Systems, Switched Observer, Stabilizability, Constant Input

\section{Introduction}

Switched system is one of the so-called hybrid systems which consist of a family of subsystems and a switching rule among them. The aspect of the switched system is found in various fields such as aircraft industry, mobile robot, animal world and Ethernet etc[3], [7].Further, the idea of switching has also been used to design intelligent control which is based on the switching between different controllers. An important problem in such switched systems is the stability problem with arbitrary switching and the stabilization problem via appropriate switching rule. Until now many results on stability and stabilization problems for various types of switched linear systems without input have been studied (e.g., [1],[2],[5], [6], [8]-[20]).

In addition, it is also important to consider the case which contains the control input for practical applications. In particular, Deaecto et al. [4] gave some conditions for some equilibrium point to be globally asymptotically stable via state feedback switched rule. The conditions are related to continuous-time switched linear system with constant input. The results were applied to DC-DC converters control design. However, the same problems via switched observer which contains information of the outputs instead of the state for the switched systems have not been investigated.

The objective of this paper is to study conditions under which equilibrium points are globally asymptotically stable via the switched observer. The conditions are related to continuous-time switched linear systems with constant input. In Section 2 the main results of this paper are given. In Section3two illustrative numerical examples are shown. Finally, concluding remarks are given in Section 4.

\section{Stabilizability Conditions}

At first, the following notations which will be needed throughout this study are given.

\section{Notations}

- $\lambda_{\max }(M)$ is the maximum eigenvalue of a symmetric matrix $M \in \mathbb{R}^{n \times n}$.

- $\|M\|$ is the maximum singular value of a matrix $M \in \mathbb{R}^{n \times m}$ (i.e., $\|M\|^{2}=\lambda_{\max }\left(M^{\mathrm{T}} M\right)$ ).

- For two matrices $M_{1}, M_{2}, M_{1}>M_{2}$ implies that $M_{1}-M_{2}$ is positive definite (i.e., $\left.M_{1}-M_{2}>0\right)$.

$-\Lambda:=\left\{\lambda=\left(\lambda_{1}, \lambda_{2}, \cdots, \lambda_{N}\right) \mid \sum_{i=1}^{N} \lambda_{i}=1, \lambda_{i} \geq 0\right\}$.

- $\quad A_{\lambda}:=\sum_{i=1}^{N} \lambda_{i} A_{i}, \lambda=\left(\lambda_{1}, \lambda_{2}, \cdots, \lambda_{N}\right) \in \Lambda$.

- $\quad \mathbb{N}:=\{1,2, \cdots N\}$.

- $\underset{i \in \mathbb{N}}{\arg \min } S:=\min \left\{i: s_{i}=\min _{j \in \mathbb{N}}\left(s_{j}\right)\right\}$ That is the minimum indexi such that $s_{i}$ is equal to the smallest element of the ordered set $S=\left\{s_{1}, s_{2}, \cdots, s_{N}\right\}$.

Next, consider the following continuous-time switched linear system 
$\Sigma_{\sigma}:\left\{\begin{array}{l}\dot{x}(t)=A_{\sigma(\hat{x}, t)} x(t)+B_{\sigma(\hat{x}, t)} u(t), x(0)=x_{0}, \\ y(t)=C_{\sigma(\hat{x}, t)} x(t),\end{array}\right.$

where $x(t) \in \mathbb{R}^{n}$ is the state, $u(t)=u \in \mathbb{R}^{m}$ is the constant input, $y(t) \in \mathbb{R}^{p}$ is the output, $\hat{x}(t) \in \mathbb{R}^{n}$ is the state of the following switched linear observer.

$$
\begin{aligned}
\frac{d}{d t} \hat{x}(t) & =A_{\sigma(\hat{x}, t)} \hat{x}(t)+L_{\sigma(\hat{x}, t)}\left\{y(t)-C_{\sigma(\hat{x}, t)} \hat{x}(t)\right\} \\
& +B_{\sigma(\hat{x}, t)} u,
\end{aligned}
$$

where $\sigma(\hat{x}, t): \mathbb{R}^{n} \times \mathbb{R}^{+} \rightarrow \mathbb{N}$ is the switching strategy which depends on the observer state $\hat{x}$ and $L_{\sigma(\hat{x}, t)}$ is the observer gain.

Now, consider the following closed-loop system $\tilde{\Sigma}_{\sigma}$ combined by the switched system $\Sigma_{\sigma}$ and the switched observer (1).

$$
\begin{aligned}
\tilde{\Sigma}_{\sigma}: \frac{d}{d t} \tilde{x}(t) & =\left[\begin{array}{cc}
A_{\sigma(\hat{x}, t)} & L_{\sigma(\hat{x}, t)} C_{\sigma(\hat{x}, t)} \\
0 & A_{\sigma(\hat{x}, t)}-L_{\sigma(\hat{x}, t)} C_{\sigma(\hat{x}, t)}
\end{array}\right] \tilde{x}(t) \\
& +\left[\begin{array}{c}
B_{\sigma(\hat{x}, t)} \\
0
\end{array}\right] u,
\end{aligned}
$$

where $\tilde{x}(t):=\left[\hat{x}^{\mathrm{T}}(t)(x(t)-\hat{x}(t))^{\mathrm{T}}\right]^{\mathrm{T}}$ is the extended state vector.

The following lemma will be used to prove our main results.

Lemma1. [14] Suppose that $\varepsilon>0, \eta>0$, $P_{1} \in \mathbb{R}^{n \times n}(>0)$ is a positive-definite matrix and $L_{\sigma} \in \mathbb{R}^{n \times p}, C_{\sigma} \in \mathbb{R}^{p \times n}$.

If $H_{\sigma}:=-P_{1} L_{\sigma} C_{\sigma}$ and

$$
\alpha>\frac{\max _{\sigma \in \mathbb{N}}\left\|H_{\sigma}\right\|^{2}}{\varepsilon \eta},
$$

then the following matrix

$$
\tilde{P}_{\sigma}:=\left[\begin{array}{cc}
\varepsilon I & -P_{1} L_{\sigma} C_{\sigma} \\
-\left(P_{1} L_{\sigma} C_{\sigma}\right)^{\mathrm{T}} & \alpha \eta I
\end{array}\right]
$$

is positive-definite.

Then, the following theorem can be obtained.

Theorem 1. Suppose that a switched system $\Sigma_{\sigma}$ with constant input $u(t)=u$ is given. Let $x_{e} \in \mathbb{R}^{n}$ be given. Suppose that the following two conditions (i) and (ii) are satisfied:

(i) There exist $\lambda \in \Lambda$, a positive-definite matrix $P_{1}(>0)$ and $\varepsilon>0$ such that

$$
\begin{aligned}
& A_{\lambda}^{\mathrm{T}} P_{1}+P_{1} A_{\lambda}<-\varepsilon I, \\
& A_{\lambda} x_{e}+B_{\lambda} u=0 .
\end{aligned}
$$

(ii)There exist a positive-definite matrix $P_{2}(>0)$ and $Y_{i} \in \mathbb{R}^{n \times p}(i=1, \cdots, N)$ such that

$$
\left\{\begin{array}{c}
A_{1}^{\mathrm{T}} P_{2}+P_{2} A_{1}-C_{1}^{\mathrm{T}} Y_{1}^{\mathrm{T}}-Y_{1} C_{1}<-\eta I \\
\vdots \\
A_{N}^{\mathrm{T}} P_{2}+P_{2} A_{N}-C_{N}^{\mathrm{T}} Y_{N}^{\mathrm{T}}-Y_{N} C_{N}<-\eta I
\end{array}\right.
$$

for some $\eta>0$.

Then $\lim _{t \rightarrow \infty} x(t)=x_{e}$ for an arbitrary initial state $x_{0} \in \mathbb{R}^{n}$ via the switching rule

$$
\sigma(\hat{x}, t)=\underset{i \in \mathbb{N}}{\arg \min } \hat{\xi} P_{1}\left(A_{i} \hat{x}+B_{i} u\right), \quad \hat{\xi}:=\hat{x}-x_{e}
$$

which depends on the state $\hat{x}$ of switched observer (1) with observer gain matrices $L_{i}:=P_{2}^{-1} Y_{i}(i=1, \cdots, N)$.

Proof. Define $\tilde{\xi}:=\left[\hat{\xi}^{\mathrm{T}}(\xi-\hat{\xi})^{\mathrm{T}}\right]^{\mathrm{T}}\left(\xi:=x-x_{e}\right)$ and a quadratic form $V(\tilde{\xi})$ as $V(\tilde{\xi})=\tilde{\xi}^{\mathrm{T}} \tilde{P} \tilde{\xi}$.Denote $H_{\sigma}:=-P_{1} L_{\sigma} C_{\sigma}$ and

$\tilde{P}:=\left[\begin{array}{cc}P_{1} & 0 \\ 0 & \alpha P_{2}\end{array}\right]$,

where $\tilde{P}$ is a positive-definite matrix. Suppose that

$$
\alpha>\frac{\max _{\sigma \in \mathbb{N}}\left\|H_{\sigma}\right\|^{2}}{\varepsilon \eta}(>0),
$$

Then, it follows from (2), (3), (4) and (5) that the time derivative of $V(\tilde{\xi})$ satisfies the following equations:

$$
\begin{aligned}
\dot{V}(\tilde{\xi})= & \dot{\tilde{x}}^{\mathrm{T}}\left[\begin{array}{cc}
P_{1} & 0 \\
0 & \alpha P_{2}
\end{array}\right] \tilde{\xi}+\tilde{\xi}^{\mathrm{T}}\left[\begin{array}{cc}
P_{1} & 0 \\
0 & \alpha P_{2}
\end{array}\right] \dot{\tilde{x}} \\
= & \left\{A_{\sigma} \hat{x}+L_{\sigma} C_{\sigma}(x-\hat{x})+B_{\sigma} u\right\}^{\mathrm{T}} P_{1} \hat{\xi} \\
& \quad+\alpha\left\{\left(A_{\sigma}-L_{\sigma} C_{\sigma}\right)(x-\hat{x})\right\}^{\mathrm{T}} P_{2}(\xi-\hat{\xi})
\end{aligned}
$$




$$
\begin{aligned}
& +\hat{\xi}^{\mathrm{T}} P_{1}\left\{A_{\sigma} \hat{x}+L_{\sigma} C_{\sigma}(x-\hat{x})+B_{\sigma} u\right\} \\
& +\alpha(\xi-\hat{\xi})^{\mathrm{T}} P_{2}\left\{\left(A_{\sigma}-L_{\sigma} C_{\sigma}\right)(x-\hat{x})\right\} \\
& =2 \hat{\xi}^{\mathrm{T}} P_{1}\left(A_{\sigma} \hat{x}+B_{\sigma} u\right)+2 \hat{\xi}^{\mathrm{T}} P_{1} L_{\sigma} C_{\sigma}(x-\hat{x}) \\
& +2 \alpha(\xi-\hat{\xi})^{\mathrm{T}} P_{2}\left(A_{\sigma}-L_{\sigma} C_{\sigma}\right)(x-\hat{x}) \\
& =2 \hat{\xi}^{\mathrm{T}} P_{1}\left(A_{\sigma} \hat{x}+B_{\sigma} u\right) \\
& +2 \hat{\xi}^{\mathrm{T}} P_{1} L_{\sigma} C_{\sigma}\left\{\left(\xi+x_{e}\right)-\left(\hat{\xi}+x_{e}\right)\right\} \\
& +2 \alpha(\xi-\hat{\xi})^{\mathrm{T}} P_{2}\left(A_{\sigma}-L_{\sigma} C_{\sigma}\right) \\
& \cdot\left\{\left(\xi+x_{e}\right)-\left(\hat{\xi}+x_{e}\right)\right\} \\
& =2 \min _{i \in \mathbb{N}}\left\{\hat{\xi}^{\mathrm{T}} P_{1}\left(A_{i} \hat{x}+B_{i} u\right)\right\} \\
& +2 \hat{\xi}^{\mathrm{T}} P_{1} L_{\sigma} C_{\sigma}(\xi-\hat{\xi}) \\
& +2 \alpha(\xi-\hat{\xi})^{\mathrm{T}} P_{2}\left(A_{\sigma}-L_{\sigma} C_{\sigma}\right)(\xi-\hat{\xi}) \\
& =\min _{i \in \mathbb{N}}\left[2 \hat{\xi}^{\mathrm{T}} P_{1}\left\{A_{i}\left(\xi+x_{e}\right)+B_{i} u\right\}\right] \\
& +2 \hat{\xi}^{\mathrm{T}} P_{1} L_{\sigma} C_{\sigma}(\xi-\hat{\xi}) \\
& +\alpha(\xi-\hat{\xi})^{\mathrm{T}}\left\{\left(A_{\sigma}-L_{\sigma} C_{\sigma}\right)^{\mathrm{T}} P_{2}\right. \\
& \left.+P_{2}\left(A_{\sigma}-L_{\sigma} C_{\sigma}\right)\right\}(\xi-\hat{\xi}) \\
& =\min _{i \in \mathbb{N}}\left\{\hat{\xi}^{\mathrm{T}}\left(A_{i}^{\mathrm{T}} P_{1}+P_{1} A_{i}\right) \hat{\xi}\right. \\
& \left.+2 \hat{\xi}^{\mathrm{T}} P_{1}\left(A_{i} x_{e}+B_{i} u\right)\right\} \\
& +2 \hat{\xi}^{\mathrm{T}} P_{1} L_{\sigma} C_{\sigma}(\xi-\hat{\xi}) \\
& +\alpha(\xi-\hat{\xi})^{\mathrm{T}}\left(A_{\sigma}^{\mathrm{T}} P_{2}+P_{2} A_{\sigma}\right. \\
& \left.-C_{\sigma}^{\mathrm{T}} Y_{\sigma}^{\mathrm{T}}-Y_{\sigma} C_{\sigma}\right)(\xi-\hat{\xi})
\end{aligned}
$$$$
<\min _{\lambda \in \Lambda}\left\{\hat{\xi}^{\mathrm{T}}\left(A_{\lambda}^{\mathrm{T}} P_{1}+P_{1} A_{\lambda}\right) \hat{\xi}\right.
$$$$
\left.+2 \hat{\xi}^{\mathrm{T}} P_{1}\left(A_{\lambda} x_{e}+B_{\lambda} u\right)\right\}
$$$$
+2 \hat{\xi}^{\mathrm{T}} P_{1} L_{\sigma} C_{\sigma}(\xi-\hat{\xi})-\alpha \eta(\xi-\hat{\xi})^{\mathrm{T}}(\xi-\hat{\xi})
$$$$
<-\varepsilon \hat{\xi}^{\mathrm{T}} \hat{\xi}+2 \hat{\xi}^{\mathrm{T}} P_{1} L_{\sigma} C_{\sigma}(\xi-\hat{\xi})
$$$$
-\alpha \eta(\xi-\hat{\xi})^{\mathrm{T}}(\xi-\hat{\xi})
$$$$
=-\left[\hat{\xi}^{\mathrm{T}}(\xi-\hat{\xi})^{\mathrm{T}}\right]\left[\begin{array}{cc}
\varepsilon I & -P_{1} L_{\sigma} C_{\sigma} \\
-\left(P_{1} L_{\sigma} C_{\sigma}\right)^{\mathrm{T}} & \alpha \eta I
\end{array}\right]
$$$$
\cdot\left[\begin{array}{c}
\hat{\xi} \\
\xi-\hat{\xi}
\end{array}\right] \cdot
$$

Since $\alpha>\frac{\max _{\sigma \in \mathbb{N}}\left\|H_{\sigma}\right\|^{2}}{\varepsilon \eta}(>0)$, it follows from Lemma1 that

$$
\tilde{P}_{\sigma}:=\left[\begin{array}{cc}
\varepsilon I & -P_{1} L_{\sigma} C_{\sigma} \\
-\left(P_{1} L_{\sigma} C_{\sigma}\right)^{\mathrm{T}} & \alpha \eta I
\end{array}\right]
$$

is positive-definite. Hence $\frac{d}{d t} V(\tilde{\xi})<0$ which implies that $V(\tilde{\xi})$ is a Lyapunov function for the extended switched system $\tilde{\Sigma}_{\sigma}$ which implies

$\lim _{t \rightarrow \infty} \hat{\xi}(t)=\lim _{t \rightarrow \infty}\left(\hat{x}(t)-x_{e}\right)=0$

and

$\lim _{t \rightarrow \infty}(\xi(t)-\hat{\xi}(t))=\lim _{t \rightarrow \infty}(x(t)-\hat{x}(t))=0$.

Thus, $\lim _{t \rightarrow \infty} x(t)=x_{e}$ for an arbitrary initial state $x_{0} \in \mathbb{R}^{n}$ via the switching rule (2).This completes the proof of Theorem1.

The following theorem says that the state $x_{e}$ is asymptotically stable via the switched rule which is linear on $\hat{x}$ if there exists a common solution $P_{1}(>0)$ satisfying Lyapunov inequalities for stable subsystems matrices $A_{i}(i=1, \cdots, N)$.

Theorem 2. Suppose that a switched system $\sum_{\sigma}$ with constant input $u(t)=u$ and $x_{e} \in \mathbb{R}^{n}$ be given. If the following two conditions (i) and (ii) are satisfied, then $\lim x(t)=x_{e}$ for an arbitrary initial state $x_{0} \in \mathbb{R}^{n}$ via the switching rule

$$
\sigma(\hat{x}, t)=\underset{i \in \mathbb{N}}{\arg \min } \hat{\xi} P_{1}\left(A_{i} x_{e}+B_{i} u\right), \hat{\xi}:=\hat{x}-x_{e}
$$

which depends on the state $\hat{x}$ of switched observer (1) with $L_{\sigma}:=P_{2}^{-1} Y_{\sigma}$.

(i) There exist $\lambda \in \Lambda$, a positive-definite matrix $P_{1}(>0)$ and $\varepsilon>0$ such that

$A_{i}^{\mathrm{T}} P_{1}+P_{1} A_{i}<-\varepsilon I$,

$A_{\lambda} x_{e}+B_{\lambda} u=0$.

(ii)There exist a positive-definite matrix $P_{2}$ (>0) and $Y_{i} \in \mathbb{R}^{n \times p}$ such that 


$$
\left\{\begin{array}{c}
A_{1}^{\mathrm{T}} P_{2}+P_{2} A_{1}-C_{1}^{\mathrm{T}} Y_{1}^{\mathrm{T}}-Y_{1} C_{1}<-\eta I \\
\vdots \\
A_{N}^{\mathrm{T}} P_{2}+P_{2} A_{N}-C_{N}^{\mathrm{T}} Y_{N}^{\mathrm{T}}-Y_{N} C_{N}<-\eta I
\end{array}\right.
$$

for some $\eta>0$.

Proof. Define $\tilde{\xi}:=\left[\hat{\xi}^{\mathrm{T}}(\xi-\hat{\xi})^{\mathrm{T}}\right]^{\mathrm{T}}\left(\xi:=x-x_{e}\right)$ and a quadratic form $V(\tilde{\xi})$ as

$$
V(\tilde{\xi})=\tilde{\xi}^{\mathrm{T}} \tilde{P} \tilde{\xi}, \quad \tilde{P}:=\left[\begin{array}{cc}
P_{1} & 0 \\
0 & \alpha P_{2}
\end{array}\right],
$$

where $\tilde{P}$ is apositive-definite matrix and

$$
\alpha>\frac{\max _{\sigma \in \mathbb{N}}\left\|H_{\sigma}\right\|^{2}}{\varepsilon \eta}(>0), \quad H_{\sigma}:=-P_{1} L_{\sigma} C_{\sigma} .
$$

Then, it follows from (6), (7), (8) and (9) that the time derivative of $V(\tilde{\xi})$ satisfies the equations:

$$
\begin{aligned}
\dot{V}(\tilde{\xi})=\dot{\tilde{x}}^{\mathrm{T}}\left[\begin{array}{cc}
P_{1} & 0 \\
0 & \alpha P_{2}
\end{array}\right] \tilde{\xi}+\tilde{\xi}^{\mathrm{T}}\left[\begin{array}{cc}
P_{1} & 0 \\
0 & \alpha P_{2}
\end{array}\right] \dot{\tilde{x}} \\
=\left\{A_{\sigma} \hat{x}+L_{\sigma} C_{\sigma}(x-\hat{x})+B_{\sigma} u\right\}^{\mathrm{T}} P_{1} \hat{\xi} \\
+\alpha\left\{\left(A_{\sigma}-L_{\sigma} C_{\sigma}\right)(x-\hat{x})\right\}^{\mathrm{T}} P_{2}(\xi-\hat{\xi}) \\
+\hat{\xi}^{\mathrm{T}} P_{1}\left\{A_{\sigma} \hat{x}+L_{\sigma} C_{\sigma}(x-\hat{x})+B_{\sigma} u\right\} \\
+\alpha(\xi-\hat{\xi})^{\mathrm{T}} P_{2}\left\{\left(A_{\sigma}-L_{\sigma} C_{\sigma}\right)(x-\hat{x})\right\} \\
=2 \hat{\xi}^{\mathrm{T}} P_{1}\left(A_{\sigma} \hat{x}+B_{\sigma} u\right)+2 \hat{\xi}^{\mathrm{T}} P_{1} L_{\sigma} C_{\sigma}(x-\hat{x}) \\
+2 \alpha(\xi-\hat{\xi})^{\mathrm{T}} P_{2}\left(A_{\sigma}-L_{\sigma} C_{\sigma}\right)(x-\hat{x}) \\
=2 \hat{\xi}^{\mathrm{T}} P_{1} A_{\sigma} \xi+2 \hat{\xi}^{\mathrm{T}} P_{1}\left(A_{\sigma} x_{e}+B_{\sigma} u\right) \\
\quad+2 \hat{\xi}^{\mathrm{T}} P_{1} L_{\sigma} C_{\sigma}\left\{\left(\xi+x_{e}\right)-\left(\hat{\xi}+x_{e}\right)\right\} \\
\quad+2 \alpha(\xi-\hat{\xi})^{\mathrm{T}} P_{2}\left(A_{\sigma}-L_{\sigma} C_{\sigma}\right) \\
\quad \cdot\left\{\left(\xi+x_{e}\right)-\left(\hat{\xi}+x_{e}\right)\right\} \\
=\hat{\xi}^{\mathrm{T}}\left(A_{\sigma}^{\mathrm{T}} P_{1}+P_{1} A_{\sigma}\right) \xi \\
+2 \min _{i \in \mathbb{N}}^{\mathrm{T}} P_{1}\left(A_{i} x_{e}+B_{i} u\right) \\
+2 \hat{\xi}^{\mathrm{T}} P_{1} L_{\sigma} C_{\sigma}(\xi-\hat{\xi}) \\
+2 \alpha(\xi-\hat{\xi})^{\mathrm{T}} P_{2}\left(A_{\sigma}-L_{\sigma} C_{\sigma}\right)(\xi-\hat{\xi}) \\
<-\varepsilon \hat{\xi}^{\mathrm{T}} \hat{\xi}+2 \min _{\lambda \in \Lambda} \hat{\xi}^{\mathrm{T}} P_{1}\left(A_{i} x_{e}+B_{i} u\right) \\
+2 \hat{\xi}^{\mathrm{T}} P_{1} L_{\sigma} C_{\sigma}(\xi-\hat{\xi}) \\
+\alpha(\xi-\hat{\xi})^{\mathrm{T}}\left\{\left(A_{\sigma}-L_{\sigma} C_{\sigma}\right)^{\mathrm{T}} P_{2}\right. \\
\left.+P_{2}\left(A_{\sigma}-L_{\sigma} C_{\sigma}\right)\right\}(\xi-\hat{\xi})
\end{aligned}
$$

$$
\begin{aligned}
& \begin{array}{r}
=-\varepsilon \hat{\xi}^{\mathrm{T}} \hat{\xi}+2 \hat{\xi}^{\mathrm{T}} P_{1} L_{\sigma} C_{\sigma}(\xi-\hat{\xi}) \\
+\alpha(\xi-\hat{\xi})^{\mathrm{T}}\left(A_{\sigma}^{\mathrm{T}} P_{2}+P_{2} A_{\sigma}\right. \\
\left.\quad-C_{\sigma}^{\mathrm{T}} Y_{\sigma}^{\mathrm{T}}-Y_{\sigma} C_{\sigma}\right)(\xi-\hat{\xi}) \\
<-\varepsilon \hat{\xi}^{\mathrm{T}} \hat{\xi}+2 \hat{\xi}^{\mathrm{T}} P_{1} L_{\sigma} C_{\sigma}(\xi-\hat{\xi}) \\
-\alpha \eta(\xi-\hat{\xi})^{\mathrm{T}}(\xi-\hat{\xi})
\end{array} \\
& =-\left[\hat{\xi}^{\mathrm{T}}(\xi-\hat{\xi})^{\mathrm{T}}\right]\left[\begin{array}{cc}
\varepsilon I & -P_{1} L_{\sigma} C_{\sigma} \\
-\left(P_{1} L_{\sigma} C_{\sigma}\right)^{\mathrm{T}} & \alpha \eta I
\end{array}\right] \\
& \cdot\left[\begin{array}{c}
\hat{\xi} \\
\xi-\hat{\xi}
\end{array}\right] .
\end{aligned}
$$

Hence, it follows from Lemma1 that $\frac{d}{d t} V(\tilde{\xi})<0$ which implies that $V(\tilde{\xi})$ is a Lyapunov function for the extended switched system $\tilde{\Sigma}_{\sigma}$. Thus, $\lim _{t \rightarrow \infty} x(t)=x_{e}$ for an arbitrary initial state $x_{0} \in \mathbb{R}^{n}$ via the switching rule (6).

\section{Illustrative Numerical Examples}

In this section, two numerical examples for Theorem1and Theorem2 will be shown.

\subsection{An example for Theorem 1}

Consider the two-dimensional switched linear system $\Sigma_{\sigma}$ with constant input which consists of two subsystems and switched observer (1). Here, each subsystem's matrices $A_{i}$, input matrices $B_{i}$ and output matrices $C_{i}$, are as follows. In this example, we note that $A_{1}$ and $A_{2}$ are unstable.

$$
\begin{aligned}
& A_{1}=\left[\begin{array}{cc}
-1 & 2 \\
2 & -1
\end{array}\right], \quad B_{1}=\left[\begin{array}{l}
3 \\
3
\end{array}\right], \quad C_{1}=\left[\begin{array}{ll}
4 & 2
\end{array}\right] \\
& A_{2}=\left[\begin{array}{ll}
-1 & -2 \\
-2 & -1
\end{array}\right], \quad B_{2}=\left[\begin{array}{l}
2 \\
1
\end{array}\right], \quad C_{2}=\left[\begin{array}{ll}
6 & 1
\end{array}\right] .
\end{aligned}
$$

Now, if we choose an input $u=1$, a positive definite matrix $P_{1}=I_{2}, \varepsilon=1 / 2$, parameters

$\lambda_{1}=0.4, \quad \lambda_{2}=0.6 \quad\left(\lambda_{1}+\lambda_{2}=1\right)$

and $x_{e}=\left[\begin{array}{ll}2 & 1\end{array}\right]^{\mathrm{T}}$, then the condition (i) of Theorem 1 is satisfied.

Next, if we choose the observer gain

$L_{1}:=P_{2}^{-1} Y_{1}, L_{2}:=P_{2}^{-1} Y_{2}$, 
where $P_{1}=I_{2}, Y_{1}:=\left[\begin{array}{l}3 \\ 2\end{array}\right], Y_{2}:=\left[\begin{array}{l}5 \\ 1\end{array}\right]$ and $\eta=1 / 8$, then the condition (ii) of Theorem 1 is also satisfied. Thus, $x_{e}$ is globally asymptotically stable via the following switched rule

$$
\sigma(\hat{x}, t)=\underset{i \in\{1,2\}}{\arg \min } \hat{\xi} P_{1}\left(A_{i} \hat{x}+B_{i} u\right) .
$$

In fact, for this example, if we choose an initial state $x_{0}=\left[\begin{array}{ll}3 & 6\end{array}\right]^{\mathrm{T}}$ of the system $\Sigma_{\sigma}$ and $x_{0}=$ [3 5.5$]^{\mathrm{T}}$ of the switched observer (1), then we have

$$
\left\{\begin{array}{l}
\hat{\xi}_{0} P_{1}\left(A_{1} \hat{x}_{0}+B_{1} u\right)=26.7500 \\
\hat{\xi}_{0} P_{1}\left(A_{2} \hat{x}_{0}+B_{2} u\right)=-59.2500
\end{array}, \hat{\xi}_{0}:=\hat{x}_{0}-x_{e} .\right.
$$

According to the switching rule $\sigma(\hat{x}, t)$ in (10), $\sigma\left(\hat{x}_{0}, 0\right)=2$ is chosen. Further, if we choose the number of subsystems according to the switching rule in the same way, then we can see the states $x(t)$ and $\hat{x}(t)$ go to $x_{e}$ as $t$ tends to $\infty$ (see Figure 1).

\subsection{An example for Theorem 2}

Consider the two-dimensional switched linear system $\Sigma_{\sigma}$ with constant input which consists of two subsystems and switched observer (1). Here, each subsystem's matrices $A_{i}$, input matrices $B_{i}$ and output matrices $C_{i}$, are as follows.

$$
\begin{array}{ll}
A_{1}=\left[\begin{array}{cc}
-2 & -1 \\
0 & -3
\end{array}\right], \quad B_{1}=\left[\begin{array}{l}
1 \\
7
\end{array}\right], \quad C_{1}=\left[\begin{array}{ll}
1 & 2
\end{array}\right] \\
A_{2}=\left[\begin{array}{cc}
-1 & 0 \\
-1 & -1
\end{array}\right], \quad B_{2}=\left[\begin{array}{l}
3 \\
2
\end{array}\right], \quad C_{2}=\left[\begin{array}{ll}
2 & 1
\end{array}\right] .
\end{array}
$$

Now, if we choose an input $u=1$, a positive definite matrix $P_{1}=I_{2}, \varepsilon=1 / 2$, parameters

$\lambda_{1}=0.2, \quad \lambda_{2}=0.8 \quad\left(\lambda_{1}+\lambda_{2}=1\right)$

and $x_{e}=\left[\begin{array}{ll}2 & 1\end{array}\right]^{\mathrm{T}}$, then the condition (i) of Theorem2 is satisfied.

Next, if we choose the observer gain $L_{1}:=P_{2}^{-1} Y_{1}, \quad L_{2}:=P_{2}^{-1} Y_{2}$,

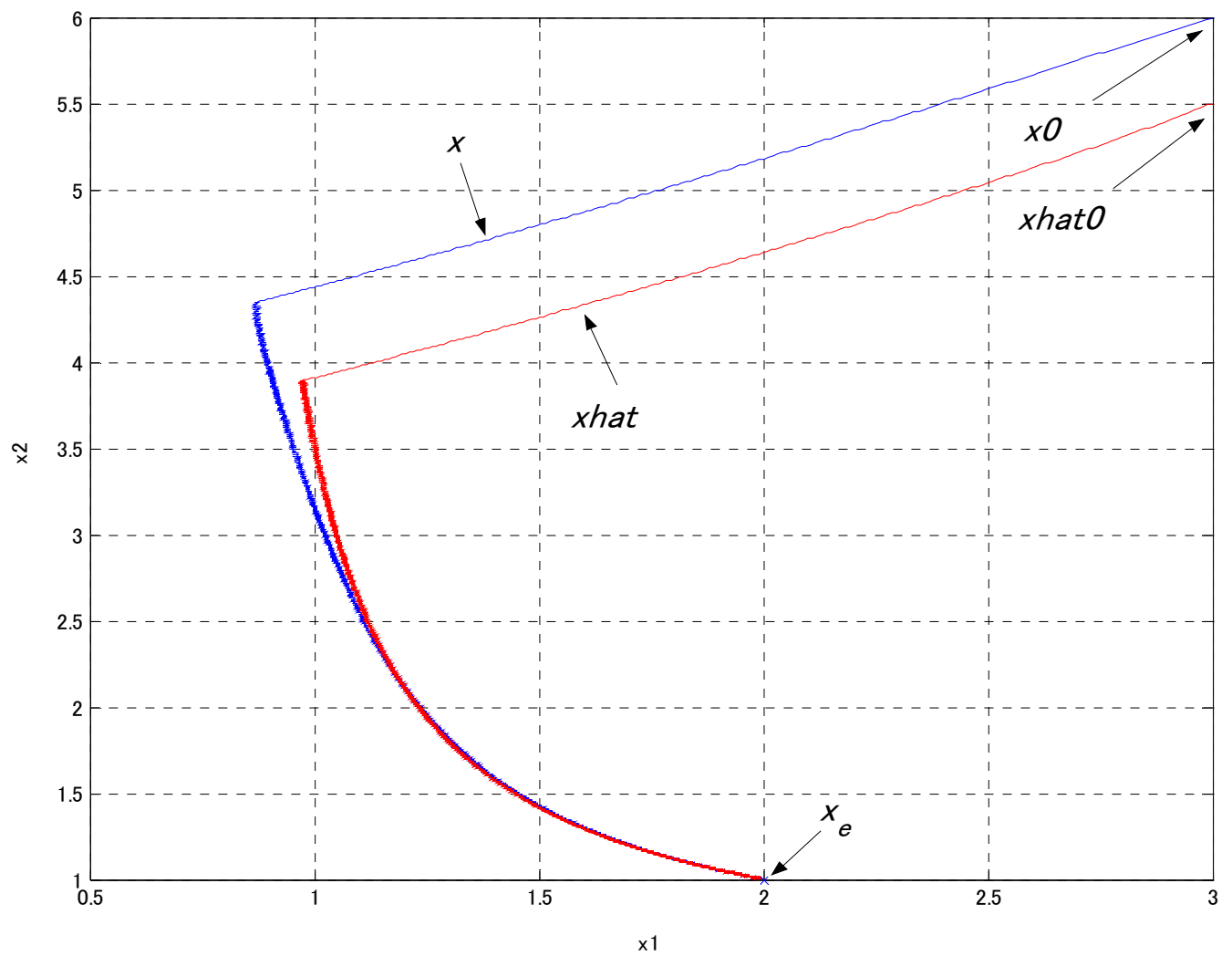

Figure 1. State trajectories of $x(t)$ and $\hat{x}(t)$ 
where $P_{1}=I_{2}, Y_{1}:=\left[\begin{array}{l}1 \\ 0\end{array}\right], Y_{2}:=\left[\begin{array}{l}3 \\ 1\end{array}\right]$ and a parameter $\eta=1$, then the condition (ii) of Theorem2 is satisfied. Thus, $x_{e}$ is globally asymptotically stable via the following switched rule

$$
\sigma(\hat{x}, t)=\underset{i \in\{1,2\}}{\arg \min } \hat{\xi} P_{1}\left(A_{i} x_{e}+B_{i} u\right) .
$$

In fact, for this example, if we choose an initial state $x_{0}=\left[\begin{array}{ll}6 & 3\end{array}\right]^{\mathrm{T}}$ of the system $\Sigma_{\sigma}$ and $x_{0}=$ $\left[\begin{array}{ll}6 & 2.7\end{array}\right]^{\mathrm{T}}$ of the switched observer (1), then we have

$$
\left\{\begin{array}{l}
\hat{\xi}_{0} P_{1}\left(A_{1} \hat{x}_{0}+B_{1} u\right)=-9.2000 \\
\hat{\xi}_{0} P_{1}\left(A_{2} \hat{x}_{0}+B_{2} u\right)=-2.3000
\end{array}, \quad \hat{\xi}_{0}:=\hat{x}_{0}-x_{e} .\right.
$$

According to the switching rule $\sigma(\hat{x}, t)$ in (11), $\sigma\left(\hat{x}_{0}, 0\right)=1$ is chosen. Further, if we choose the number of subsystems according to the switching rule in the same way, then we can see the states $x(t)$ and $\hat{x}(t)$ go to $x_{e}$ as $t$ tends to $\infty$ (see Figure 2).

\section{Concluding Remarks}

In this paper, stabilizability for continuous-time switched linear systems with constant input via switched observer was investigated. Firstly, the conditions for equilibrium points related to the switched linear system with constant input to be globally asymptotically stable via switched observer were presented. Next, two numerical examples to illustrate the main results (Theorem 1 and Theorem 2) were also shown, respectively. As future studies we need to investigate parameter insensitive stabilization problems for switched linear systems, with constant input via switched observer.

\section{Acknowledgement}

This work was supported in part by JSPS KAKENHI Grant-in-Aid for Scientific Research(C)-22560453.

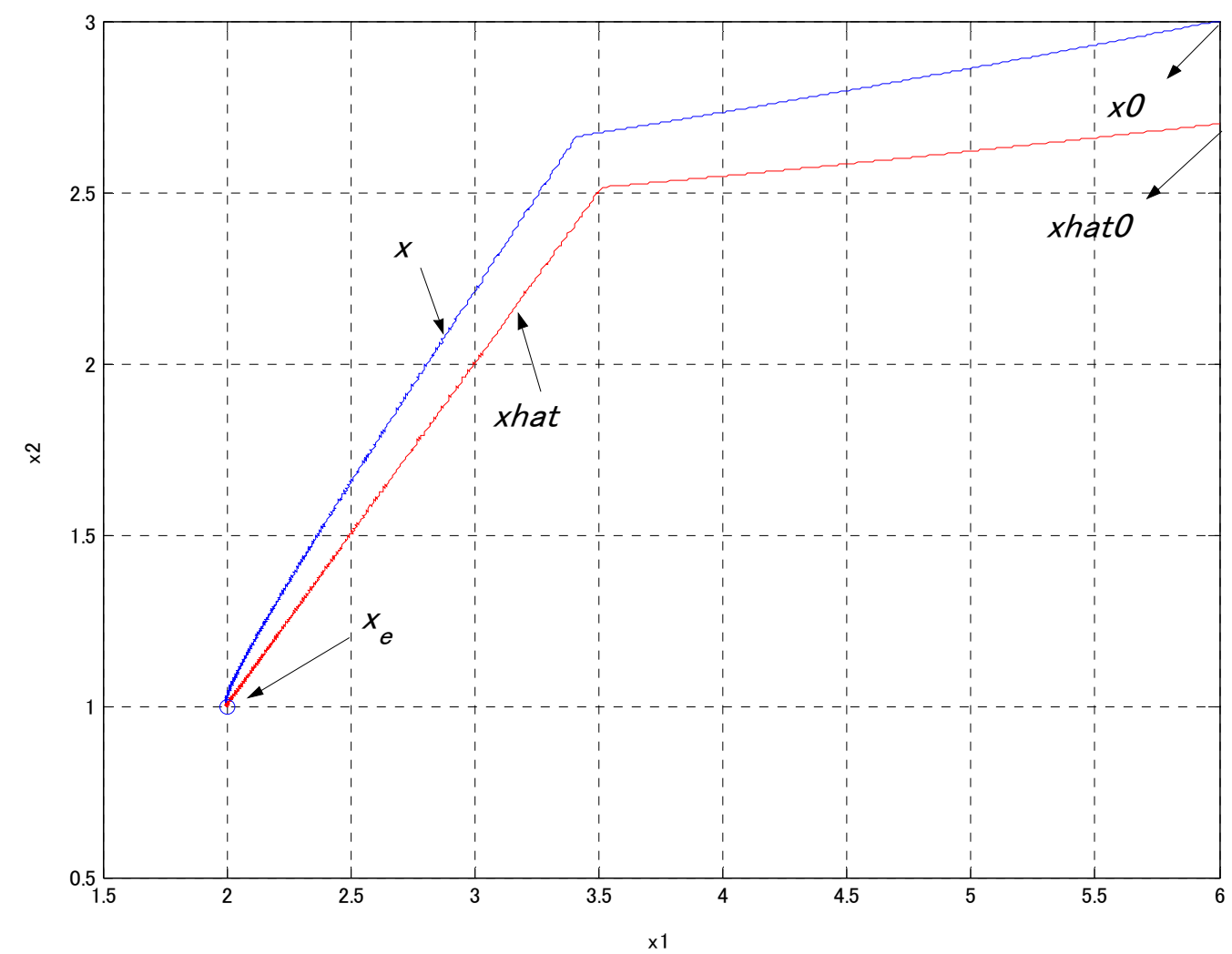

Figure 2. State trajectories of $x(t)$ and $\hat{x}(t)$ 
1. BRANICKY, M. S., Stability of Switched and Hybrid Systems, Proceedings of the 33rd IEEE Conference on Decision and Control, 1994, pp. 3498-3503.

2. BRANICKY, M. S., Multiple Lyapunov Functions and Other Analysis Tools for Switched and Hybrid Systems, IEEE Transitions on Automatic Control, vol.43, no. 4, 1998, pp. 475-482.

3. DAYAWANSA, W. P., C. F. MARTIN, A Converse Lyapunov Theorem for a Class of Dynamical Systems which Undergo Switching, IEEE Transitions on Automatic Control, vol. 44, no. 4, 1999, pp. 751-760.

4. DEAECTO, G. S., J. C. GEROMEL, F. S. GARCIA, J. A. POMILIO, Switched Affine Systems Control Design with Application to DC-DC Converters, IET Control Theory and Applications, vol. 4, no. 7, 2010, pp. 1201-1210.

5. FERON, E., Quadratic Stabilizibility of Switched Systems Via State and Output Feedback, Massachusetts Institute of Technology Technical report CICSP-468, 1996, pp. 1-13.

6. GEROMEL, J. C., P. COLANERI, P. BOLZERN, Dynamic Output Feedback Control of Switched Linear Systems, IEEE Transcriptions on Automatic Control, vol. 53, no. 3, 2008, pp.720-733.

7. KIM, M. K., L. SHAN, EDF-based Realtime Message Scheduling of Periodic Messages on a Master-Slave-based Synchronized Switched Ethernet, International Journal of Control and Automation, vol. 2, no. 4, 2009, pp. 25-34.

8. LIBERZON, D., Basic Problems in Stability and Design of Switched Systems, IEEE Control Systems Magazine, vol. 19, 1999, pp. 59-70.

9. LIBERZON, D., Switching in Systems and Control, Systems \& Control: Foundation \& Applications, Birkhäuser, 2003.
10. LIN, H., P. J. ANTSAKLIS, A Necessary and Sufficient Condition for Robust Asymptotic Stabilizability of Continuous-time Uncertain Switched Linear Systems, Proceedings of the 43rd IEEE Conference on Decision and Control, 2004, pp. 3690-3695.

11. OTSUKA, N., T. SOGA, Quadratic Stabilizability for Polytopic Uncertain Continuous-time Switched Linear Systems Composed of Two Subsystems, International Journal of Control and Automation, vol. 3, no. 1, 2010, pp. 35-42.

12. SAVKIN, A. V., R. J. EVANS, Hybrid Dynamical Systems: Controller and Sensor Switching Problems, Birkhäuser, 2002.

13. SOGA, T., N. OTSUKA, Quadratic Stabilizability for Polytopic Uncertain Continuous-time Switched Linear Systems by Output Feedback, Proceedings of the 2010 American Control Conference, 2010, pp. 3920-3925.

14. SOGA, T., N. OTSUKA, Quadratic Stabilizability for Polytopic Uncertain Continuous-time Switched Linear Systems via Switched Observer, Proceedings of the 19th Mediterranean Conference on Control and Automation, 2011, pp. 724-729.

15. SUN, Z. D., S. S. GE, Stability Theory of Switched Dynamical Systems, SpringerVerlag, 2011.

16. WANG, Y., Z. ZUO, Onquadratic Stabilizability of Linear Switched Systems with Polytopic Uncertainties, Proceedings of the 2005 IEEE International Conference on Systems, Man and Cybernetics, 2005, pp. 1640-1644.

17. WICKS, M., P. PELETIES, R. DECARLO, Construction of Piecewise Lyapunov Functions for Stabilizing Switched Systems, Proceedings of the 33rd IEEE Conference on Decision and Control, 1994, pp. 3492-3497. 
18. WICKS, M., P. PELETIES, R. DECARLO, Switched Controller Synthesis for the Quadratic Stabilization of a Pair of Unstable Linear Systems, European Journal of Control, vol.4, no.2, 1998, pp. 140-147.

19. ZHAI, G., Quadratic Stablizibility of Discrete-time Switched Systems Via State and Output Feedback, Proceedings of the 40th IEEE Conference on Decision and Control, 2001, pp. 2165-2166.
20. ZHAI, G., H. LIN, P. J. ANTSAKLIS, Quadratic Stabilizability of Switched Systems with Polytopic Uncertainties, International Journal of Control, vol. 76, no. 7, 2003, pp. 747-753. 